\title{
Effect of humanistic nursing on mental health of patients
}

\author{
Huang Yan, Chen Xiaomei \\ Chengdu Medical College, Szechwan Chengdu 610500
}

Keywords: humanistic care; Behavior pattern; mental health; SCL-90; SCSQ

\begin{abstract}
Objective: To investigate the effect of humanistic nursing on the mental health of the patients. Methods: a total of 249 patients were investigated by SCL-90 and SCSQ, of which 97 elderly patients were the experimental group, and the remaining 152 were the other patients without humanistic nursing care. Results SPSS17.0 was used for statistical analysis and comparison between groups. Results: before the implementation of humanistic nursing, there was no significant difference between the experimental group and the control group on SCL-90, P > 0.05. After the experiment, there were significant differences between the two groups in the depression items $(t=2.79, P=0.047<0.05)$, but there was no significant difference in the other 8 items, and the result was $\mathrm{P}>0.05$. The two group of the first SCSQ integral mean + SD respectively is: $1.91+0.73,1.85$ +0.65 , $\mathrm{t}=1.27$; two $\mathrm{P}=0.211>0.05$; group second SCSQ respectively: $1.44+0.52,1.82+0.63$, $\mathrm{t}=5.61 ; \mathrm{P}=0.019<0.05$, with significant difference. Conclusion: humanistic nursing has limited effect on improving the mental health of the patients, but it can significantly improve the enthusiasm of the patients. Humanistic care should be introduced into the theory and method of psychology, so as to improve the effect of humanistic nursing.

Mental health is of positive significance to the rehabilitation of patients with disease[1-5]. At the same time, it is also of great value in improving the patients' compliance with medical treatment[6-10]. And it is of great value in promoting the harmonious relationship between doctors and patients[11-17]. Our department is the first one to explore and implement the humanistic care measures that is in accordance with the western region, and has some work experience at present. Humanistic nursing care can significantly improve patients' cooperation with doctors and nurses, and promote the doctor-patient mutual trust and reduce doctor-patient disputes. In order to further study the positive impact of humanistic care on patients and provide evidence for scientific decision-making, we made a comparative study on the change of patients' mental health. From March 2017 to August 2017, 249 patients were investigated using the Symptom Checklist-90 (SCL-90) and the Simplified Coping Style Questionnaire (SCSQ). Now,we report as follows.
\end{abstract}

\section{Objects and methods}

\subsection{Objects}

249 patients from our hospital. There were 97 patients from the Department of Geriatrics with humanistic care, 55 men and 42 women, with an average year of $56.3+5.4$ years, which was the experimental group. In addition, the control group was consisted of 152 patients, 83 males and 69 females. They were from the Department of internal medicine and surgery and the average age was $53.73+10.5$. There was no significant difference between the two groups in terms of age and gender ( $P>0.05)$, so it is comparable.

\subsection{Humanistic nursing care methods}

Through practical research, our department has formed our own humanistic care measures. We hired a flight attendants professionals to set up standards of smile service, etiquette service standards for us. We strengthen communication and greetings with patients, care about patients' individual moods and troubles, listen appropriately to patients and support them; using Empathy Communication Skills (the technology that treats a patient as a relative or friend of ourselves); Treatment techniques for common complaints from patients and their families; Ask the nurse to do at least one thing for the patient to make the patient happy, even if a joke is told. 


\subsection{Methods}

The questionnaire mainly used symptom checklist [18] (SCL-90) and simple coping style questionnaire [19] (SCSQ). SCL-90 mainly contains two surveys and data collections in the test group such as somatization, obsessive-compulsive symptoms, interpersonal sensitivity, depression, anxiety, hostility, terror, paranoia and psychosis. All patients completed the questionnaire survey with the help of family members and medical staff, all of them were efficiently recycled. The investigation time was completed within the first 4 days of admission to the patients, and the second time was half a month after that, a total of two investigations was implemented. Among them, the experimental group of the Department of geriatric medicine has always been humanistic cared(The patients who were not the subjects of the investigation were humanistic cared without distinction), and the practitioners are nurses, followed by the attending physician. SPSS 17 was used for statistical t test in the questionnaire survey, and $P<0.05$ was statistically significant.

\section{Results}

\subsection{The comparison of SCL-90 points in the first time}

There was no significant difference between the experimental group and control group on the whole project, and $P>0.05$. Overall, both groups scored higher on somatization, obsessive-compulsive disorder, depression, anxiety and horror. The details are shown in Table 2.2

\subsection{The comparison of SCL-90 points in the second time There was a significant difference}

Between the two groups only in depression $(t=2.79 ; P=0.047<0.05)$, but no significant difference was found in the other 8 items. The test result was $P>0.05$. Look at Table 2 for details.

\subsection{The comparison of SCSQ points between the experimental group and the control group}

The mean \pm SD of SCSQ scores for the first time between the two groups were: $1.91 \pm 0.73$, $1.85 \pm 0.65, t=1.27 ; P=0.211>0.05$. The scores of the second SCSQ between the two groups were $1.44 \pm 0.52,1.82 \pm 0.63, t=5.61, P=0.019<0.05$, with significant differences. See Table 3.

Table 1 Comparison of the factors of the first SCL-90 between the experimental group and the control group(mean \pm SD)

\begin{tabular}{|c|c|c|c|c|c|c|c|}
\hline $\begin{array}{r}\text { groups } \\
\text { Paranoid }\end{array}$ & \multicolumn{7}{|c|}{ Psychosis } \\
\hline experimenta & l 97 & $2.2 \pm 0.5$ & $2.0 \pm 0.4$ & $1.8 \pm 0.4$ & $2.4 \pm 0.6$ & $2.3 \pm 0.6$ & $1.3 \pm 0.3$ \\
\hline $1.9 \pm 0.4$ & $1.6 \pm 0.4$ & $1.5 \pm 0.4$ & & & & & \\
\hline Control & 152 & $2.1 \pm 0.3$ & $2.1 \pm 0.5$ & $1.8 \pm 0.5$ & $2.5 \pm 0.7$ & $2.2 \pm 0.5$ & $1.4 \pm 0.4$ \\
\hline $1.8 \pm 0.4$ & $1.7 \pm 0.4$ & $1.6 \pm 0.5$ & & & & & \\
\hline$T$ value & & 1.17 & -1.02 & -0.75 & -1.22 & 0.61 & -1.24 \\
\hline 1.39 & -1.51 & -1.41 & & & & & \\
\hline Pvalue & & 0.271 & 0.119 & 0.415 & 0.267 & 0.06 & 0.214 \\
\hline 0.072 & 0.313 & 0.193 & & & & & \\
\hline
\end{tabular}

Table 2 Comparison of the factors of second times SCL-90 between the experimental group and the control group.(mean \pm SD)

\begin{tabular}{|c|c|c|c|c|c|c|c|}
\hline $\begin{array}{r}\text { groups } \\
\text { Paranoid } \\
\end{array}$ & \multicolumn{7}{|c|}{ Psychosis } \\
\hline experiment & al 97 & $1.9 \pm 0.5$ & $1.9 \pm 0.7$ & $1.8 \pm 0.6$ & $1.8 \pm 0.5$ & $2.0 \pm 0.5$ & $1.4 \pm 0.3$ \\
\hline $1.8 \pm 0.5$ & $1.7 \pm 0.4$ & $1.5 \pm 0.4$ & & & & & \\
\hline Control & 152 & $2.0 \pm 0.4$ & $2.0 \pm 0.5$ & $1.7 \pm 0.5$ & $2.2 \pm 0.6$ & $2.1 \pm 0.7$ & $1.3 \pm 0.4$ \\
\hline $1.8 \pm 0.5$ & $1.6 \pm 0.5$ & $1.6 \pm 0.5$ & & & & & \\
\hline$t$ value & & -0.121 & -1.22 & 1.03 & -2.79 & -1.54 & 1.01 \\
\hline 0.77 & 0.402 & -0.674 & & & & & \\
\hline
\end{tabular}




\begin{tabular}{cccccccc}
\hline$P$ value & & 0.178 & 0.924 & 1.02 & 0.047 & 0.122 & 0.357 \\
0.121 & 0.616 & 0.184 & & & & & \\
\hline
\end{tabular}

Table 3 Comparison of two SCSQ scores in two groups of nurses(mean \pm SD)

\begin{tabular}{lccr}
\hline \multicolumn{2}{c}{ groups $\begin{array}{c}\text { experimental,first time } \\
\text { time control,second time }\end{array}$} & control,first time & \multicolumn{2}{c}{ experimental,second } \\
\hline $\begin{array}{l}\text { Number of people } n \\
152 \quad 97\end{array}$ & 152 & 97 \\
$\begin{array}{c}\text { points } \\
1.82 \pm 0.63\end{array}$ & $1.91 \pm 0.73$ & $1.85 \pm 0.65$ & $1.44 \pm 0.52$ \\
\hline$t \quad$ value & & & 1.27 \\
$5.61 \quad$ value & & 0.211 \\
0.019 P & & \\
\hline
\end{tabular}

\section{Discussion}

\subsection{A single solution to patients with mental problems is not obvious}

The findings of this survey were surprising. It is thought that the nurses' humanistic care can effectively improve the mental health level of the patients, but the effect is not obvious in fact. Patients admitted to the hospital had their own concerns and fears about illness. Some of them also had anxiety about spending money in treatment. So the psychological problems of patients were rather complicated. The single method of nurse humanistic care may promote the harmony of the relationship between nurses and patients and enhance the compliance of patients, but it has a relatively weak impact on mental health. The effect of the single method is not good, which suggesting that the psychological problems of our patients are often difficult to deal with, and the source of their psychological problems is more complex. Meanwhile, we can not ignore the psychological problems of patients, because mental health promotion can effectively promote the rehabilitation of the disease, and rehabilitation may be able to essentially improve the patient's psychological situation.

\subsection{Humanistic care can significantly improve the patient's ability to respond positively}

The results of this study show that although nurses' humanistic care can not fundamentally improve their psychological problems, they can affect the enthusiasm of patients. There were significant differences between the experimental group and the control group, indicating the importance of humanistic care. Nurses actively interact with patients, care for patients, not only solve problems for patients, but also conduct scientific guidance for patients' life and treatment from a medical perspective, so that patients trust nurses, cooperate with treatment, and their behaviors show good enthusiasm. The main purpose of our department to explore the implementation of humanistic care is to coordinate the nurse-patient relationship and doctor-patient relationship to make patients more receptive to treatment and promote the satisfaction of patients in our department. This measure can obviously improve the enthusiasm of patients' behavior, which is an accident harvest. It further proves the importance and necessity of implementing humanistic care for patients.

\subsection{The improvement of humanistic care and nursing ability is inseparable from psychology}

The development of each work will have advantages and disadvantages, and how to further improve the nursing care can not be separated from psychology. The findings of this survey prompt us that progress in nursing humanistic care should be combined with psychology and multidisciplinary synergy to show better results. First of all, we should strengthen the training of psychological knowledge of nursing staff to master common psychological characteristics of patients and their treatment strategies. Secondly, to encourage nurses to learn psychology, obtain a psychotherapist qualification certificate, and strengthen the psychological nursing ability of nurses. Third, psychological professionals can be invited to conduct collective guidance to patients in the 
Department, while some patients with prominent psychological and behavioral problems can be provided individualized help. Fourth, we must earnestly improve the living and entertainment conditions of the wards, especially the control of the TV contents of the wards, and try to broadcast healthy, progressive, inspirational and humorous videos as well as music suitable for rest and recuperation. Improving the patient's psychological situation and mental health in many ways .

In a word, there are many positive values for humanistic care to patients. By constantly improving the means of humanistic care, we can make it a great deal. It is playing a more and more important role in improving the relationship between nurses and patients, improving the patient's compliance, and improving the mental health of the patients.

\section{Acknowledgements}

This work is supported by Scientific research project "Qualitative research on the present application of humanistic care ability of nursing staff in Chengdu City" of the Education Department of Sichuan in 2017, Number: 17SB0110.

\section{Reference}

[1]Ma Xiaoqian, Liu Shiyan, Luo Xingwei. Application and Progress of Multiple Family Therapy Model of Mental Health Education in Severe Mental Illness[J]. Chinese Journal of Clinical Psychology.2015,23(2):372-377

[2]Zhen Tianxu, Zheng Xin. Research on Effect of Mental Health Intervention on Health Promotion of Patients with Cardiovascular Diseases[J]. Journal pf Integrative Medicine Cardiovascular Diseases. 2016,10(18): 94

[3]Fan Qicai, Zhu Sanling, Zuo Yunhai. Study on the Relationship between Quality of Life and Mental Health in Patients with Cardiovascular Disease[J]. Journal of Modern Diagnosis and Therapy. 2015, 26(17): 4054-4055

[4]Yang Shujing. Interactions between Gut Microbiota and Human Diet, Mental Health and Physical Illnesses[J]. Journal of Chongqing Medical University.2017,46(1):396-399

[5]Yang Yanqiu, Wang Chengdang. Study on the Correlation between Inflammatory Bowel Disease and Mental Health[J]. Chinese Journal of digestion.. 2017,37(3):209-212

[6]Hu Yuanfeng, Gao Hongmei,Qiu Weiqin. Effect of Clinical Nursing Pathway on Compliance in Patients with Chronic Renal Failure[J]. Journal of Gilin Medical. 2014,35(15):3360-3361

[7]Zhang Ting. Effect of Nursing Intervention on the Treatment Compliance and Quality of Life in Patients with Lupus Erythematosus[J]. Shenzhen Journal of Integrated Traditional Chinese and Western Medicine.2015,25(8):154-155

[8]Shen Juan. Effect of Nursing Intervention on Compliance and Quality of Life in Chronic Hepatitis B Patients[J]. World Latest Medicine Information. 2016.15(2):323-

[9]Sun Yaping. The Effect of Quality Nursing Intervention on Compliance and Nursing Satisfaction of Elderly Patients with Hypertension[J]. Journal of chronic diseases.2016,24(10):1172-1174

[10]Liang Zhenfeng, Zhang Dongying. Effect of Psychological Nursing on Treatment Compliance in Elderly Hypertensive Patients[J]. Journal of Massage and Rehabilitation Medicine. 2017,8(10):41-42

[11]Li Li. The Effect of Humanistic Care on Improving the Relationship between Nurses and Patients in Obstetrics and Gynecology[J]. Chinese Journal of Health Standards Management.2017,8(14):169-171

[12]Zhou Lisha, Wang Jihong. Influence of Humanistic Concern on the Relationship between Nurses and Patients in Cancer Patients[J]. General Nursing Journal.2016,14(33): 3531-3531 
[13]Duan Dan, Wan Changxiu.The Role of Humanistic Care in Building Harmonious Relationship between Nurses and Patients[J].General Nursing Journal.2017,15(4):471-474

[14]Ling Jiwei,Liu Xusheng. Humanistic Medicine on the Road of Doctor-patient Relationship[J]. Journal of Medical Contention.2015,6(4):16-19

[15]Wang Yuan, Wu Xiaowei,Huang Jidong. Doctor - Patient Relationship and Cultivation of Humanistic Quality of Medical Students[J]. Chinese Medicine Guides.2017,14(19):133-136

[16]Wu Jingna, Li Xing. Reflections on the Relationship between Doctor and Patient from the Perspective of Medical Humanities[J]. Jiangsu Healthcare Administration. 2015,26(2):157-158

[17]Zhu Haixia, Xie Caiyun. Constructing Harmonious Relationship Between Doctors and Patients and Improving Humanistic Quality[J]. China Health Industry.2017,14(4):183-184

[18]Wang Xiangdong, Wang Xilin, etc. Mental Health Rating Scale Manual[J]. Chinese Journal of mental health. 1999 Supplement:33

[19]Wang Xiangdong, Wang Xilin, etc. Mental Health Rating Scale Manual[J]. Chinese Journal of mental health. 1999 Supplement:122 